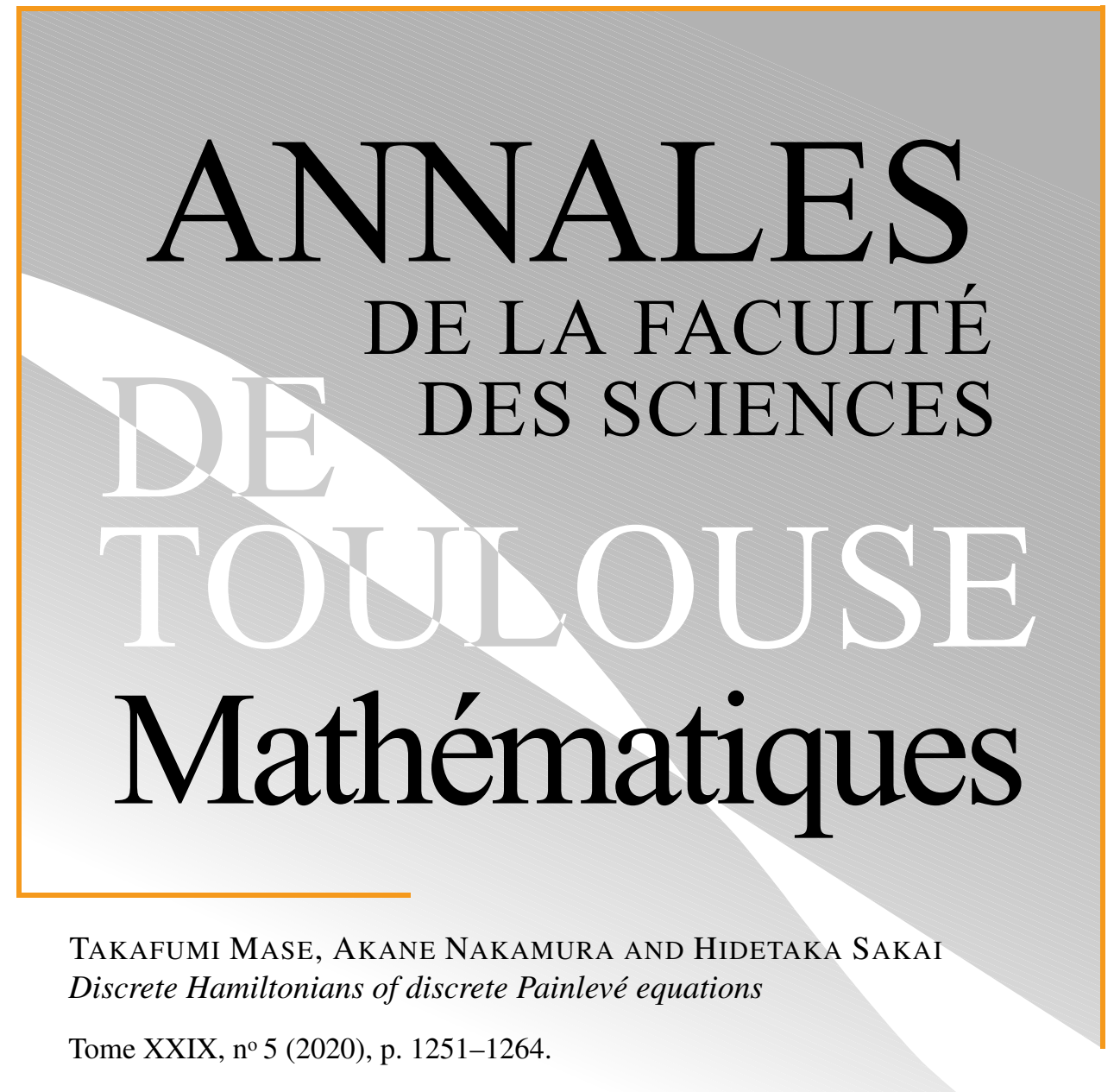

https://doi.org/10.5802/afst.1660

(C) Université Paul Sabatier, Toulouse, 2020.

L'accès aux articles de la revue «Annales de la faculté des sciences de Toulouse Mathématiques » (http://afst.centre-mersenne.org/) implique l'accord avec les conditions générales d'utilisation (http://afst.centre-mersenne.org/legal/). Les articles sont publiés sous la license CC-BY 4.0.

(c) $\underset{B Y}{(1)}$

Publication membre du centre

Mersenne pour l'édition scientifique ouverte MERSENNE http://www.centre-mersenne.org/ 


\title{
Discrete Hamiltonians of discrete Painlevé equations
}

\author{
Takafumi Mase ${ }^{(1)}$, Akane Nakamura ${ }^{(2)}$ and Hidetaka Sakai ${ }^{(3)}$
}

\begin{abstract}
We express discrete Painlevé equations as discrete Hamiltonian systems. The discrete Hamiltonian systems here mean the canonical transformations defined by generating functions. Our construction relies on the classification of the discrete Painleve equations based on the surface-type. The discrete Hamiltonians we obtain are written in the logarithm and dilogarithm functions.

RÉSumé. - Nous exprimons des équations de Painlevé discrètes sous forme de systèmes hamiltoniens discrets. Les systèmes hamiltoniens discrets désignent ici les transformations canoniques définies par la génération de fonctions. Notre construction est basée sur la classification d'équations discrètes de Painlevé basées sur le type de surface. Les hamiltoniens discrets que nous obtenons sont écrits dans les fonctions logarithme et dilogarithme.
\end{abstract}

\section{Introduction}

At the beginning of the 20th century, P. Painlevé and B. Gambier classified second order ordinary differential equations of normal form that possess the so-called Painlevé property $[1,6]$. They discovered six new transcendental equations, which are known today as the Painlevé equations. About 80 years later, singularity confinement has been proposed as a discrete analogue of the Painlevé property [3] and, with the help of this test, discrete analogues of the Painlevé equations have been discovered [8]. Today, a large number

Keywords: Integrable system, Painlevé equations.

2010 Mathematics Subject Classification: 33E17, 34M55, 39A12.

(1) Graduate School of Mathematical Sciences, University of Tokyo, Japan mase@ms.u-tokyo.ac.jp

(2) Department of Mathematics, Faculty of Science, Josai University, Japan a-naka@josai.ac.jp

(3) Graduate School of Mathematical Sciences, University of Tokyo, Japan sakai@ms.u-tokyo.ac.jp

This work is supported by Japan Society for the Promotion of Sciences (JSPS), through JSPS grants number 18K03323 and 18K13438. 
of discrete Painlevé equations are known, but most of them have been constructed by deautonomizing QRT mappings [2, 5]. Since QRT mappings can be solved by elliptic functions [7], this deautonomization procedure is parallel to that in the continuous case, where the (continuous) Painlevé equations can be thought of as deautonomized systems of ordinary differential equations of elliptic functions. Using a specific type of rational surfaces, one of the present authors classified (and in a sense, defined) discrete Painlevé equations [9]. According to this classification, the discrete Painlevé equations consist of 19 classes depending on the surface type, which we will see later. The surface associated to an equation is called the space of initial conditions and, through the theory of spaces of initial conditions, both discrete and continuous Painlevé equations, including their relations, are well-studied. See a concise review paper [4] for more information on the discrete Painlevé equations.

These days, research on discrete Painlevé equations is performed almost in parallel with research on the continuous Painlevé equations, such as the reduction to the compatibility conditions of linear equations (Lax pair), the calculation of special solutions, and so on. One of the biggest differences is that, while the Painlevé differential equations are all expressed as Hamiltonian systems, such a description in the discrete case was not yet known. Let us take a look at the Hamiltonian functions of the Painlevé differential equations:

$$
\begin{aligned}
& H_{\mathrm{VI}}\left(\begin{array}{l}
a_{1}, a_{2} \\
a_{3}, a_{4}
\end{array} ; ; q, p\right)=q(q-1)(q-s) p^{2} \\
& +\left\{\left(a_{1}+2 a_{2}\right) q(q-1)+a_{3}(s-1) q+a_{4} s(q-1)\right\} p \\
& +a_{2}\left(a_{1}+a_{2}\right) q, \quad \frac{\mathrm{d} s}{\mathrm{~d} t}=s(s-1), \\
& H_{\mathrm{V}}\left(\begin{array}{c}
a_{1}, a_{2} \\
a_{3}
\end{array} ; t ; q, p\right)=p(p+1) q\left(q+e^{t}\right)+a_{1} q(p+1)+a_{3} p q-a_{2} e^{t} p, \\
& H_{\mathrm{III}}\left(D_{6}\right)\left(a_{1}, b_{1} ; t ; q, p\right)=p(p+1) q^{2}-a_{1} p(q-1)-b_{1} p q-e^{t} q \text {, } \\
& H_{\mathrm{III}}\left(D_{7}\right)\left(a_{1} ; t ; q, p\right)=p^{2} q^{2}+a_{1} q p+e^{t} p+q, \\
& H_{\mathrm{III}}\left(D_{8}\right)(t ; q, p)=p^{2} q^{2}+q p-q-\frac{e^{t}}{q}, \\
& H_{\mathrm{IV}}\left(a_{1}, a_{2} ; t ; q, p\right)=p q(p-q-t)-a_{2} p-a_{1} q \text {, } \\
& H_{\mathrm{II}}\left(a_{1} ; t ; q, p\right)=p\left(p-q^{2}-t\right)-a_{1} q, \\
& H_{\mathrm{I}}(t ; q, p)=p^{2}-q^{3}-t q .
\end{aligned}
$$


Expressing an equation as a Hamiltonian system has many advantages. One of the most important benefit is that the Hamiltonian function automatically becomes a conserved quantity if it is autonomous. In the case of the Painlevé equations, however, this does not hold since the systems are non-autonomous. Another important advantage is that using a Hamiltonian system we can write an equation concisely. For example, this can be of help when one considers the problem of identifying equations. Since the time evolution is determined by a single function, one can compare the Hamiltonian functions instead of the time evolution equations themselves.

Roughly speaking, when a discrete dynamical system is "easily" expressed by a single function $W$ on some phase space, we call $W$ a "discrete Hamiltonian" of the system. As an example of such a function, we already know what is called the generating function of a canonical transformation.

A canonical transformation on a phase space with a symplectic structure is defined as a transformation of the Hamiltonian system that preserves the symplectic form. It is known that each canonical transformation can be written with a function $W=W(q, \bar{p})$ as

$$
p_{k}=\frac{\partial W}{\partial q_{k}}, \quad \bar{q}_{k}=\frac{\partial W}{\partial \bar{p}_{k}}, \quad k=1, \ldots, n,
$$

where $W$ is called the generating function. In the case of discrete Painlevé equations, however, it is usually more important to write a system as a birational mapping than to write in canonical variables. Therefore, in this paper, we sometimes give priority to choosing good variables over writing discrete Hamiltonians or equations in canonical variables

Remark 1.1. - Let us take a look at a relation with the Lagrangian form of discrete dynamical systems by Veselov [10]. Given a Lagrange function $L_{k}(r, s): X \times X \rightarrow \mathbb{C}$, the variation of the formal sum $S(\lambda)=$ $\sum_{k \in \mathbb{Z}} L_{k}\left(\lambda_{k}, \lambda_{k+1}\right)$

$$
\begin{aligned}
\delta S(\lambda) & =\sum_{k \in \mathbb{Z}} \delta L_{k}\left(\lambda_{k}, \lambda_{k+1}\right) \\
& =\sum_{k \in \mathbb{Z}}\left\{L_{k}\left(\lambda_{k}+\delta \lambda_{k}, \lambda_{k+1}+\delta \lambda_{k+1}\right)-L_{k}\left(\lambda_{k}, \lambda_{k+1}\right)\right\} \\
& =\sum_{k \in \mathbb{Z}}\left\{\frac{\partial L_{k}}{\partial r}\left(\lambda_{k}, \lambda_{k+1}\right) \delta \lambda_{k}+\frac{\partial L_{k}}{\partial s}\left(\lambda_{k}, \lambda_{k+1}\right) \delta \lambda_{k+1}\right\} \\
& =\sum_{k \in \mathbb{Z}}\left\{\frac{\partial L_{k}}{\partial r}\left(\lambda_{k}, \lambda_{k+1}\right)+\frac{\partial L_{k-1}}{\partial s}\left(\lambda_{k-1}, \lambda_{k}\right)\right\} \delta \lambda_{k}=0
\end{aligned}
$$


gives the discrete Euler-Lagrange equation

$$
\frac{\partial L_{k}}{\partial r}\left(\lambda_{k}, \lambda_{k+1}\right)+\frac{\partial L_{k-1}}{\partial s}\left(\lambda_{k-1}, \lambda_{k}\right)=0
$$

This equation is a second-order single equation. Let us rewrite it into a simultaneous form. Putting $\mu_{k}=\frac{\partial L_{k}}{\partial r}\left(\lambda_{k}, \lambda_{k+1}\right)=-\frac{\partial L_{k-1}}{\partial s}\left(\lambda_{k-1}, \lambda_{k}\right)$ and $W(\lambda, \bar{\mu})=\bar{\lambda} \bar{\mu}+L(\lambda, \bar{\lambda})$, we can write the equation as

$$
\bar{\lambda}=\frac{\partial W}{\partial \bar{\mu}}, \quad \mu=\frac{\partial W}{\partial \lambda},
$$

which is an expression as a canonical transformation by the generating function $W$. In fact, the symplectic form $\mathrm{d} \mu \wedge \mathrm{d} \lambda$ is preserved under this transformation. However, in order to calculate $W$ as a function in $\lambda$ and $\bar{\mu}$, we need to solve an implicit function. A similar problem occurs when one computes $L(\lambda, \bar{\lambda})$ from $W(\lambda, \bar{\mu})$.

In this paper, we do not consider the Lagrangian form but focus on generating functions of canonical transformations.

It is known that each discrete Painlevé equation can be formulated as a discrete dynamical system determined by a Cremona isometry of infinite order on a generalized Halphen surface [9]. Generalized Halphen surfaces are classified according to the type of the anti-canonical divisor. The list of the surfaces is as in Table 1.1.

Table 1.1. List of generalized Halphen surfaces

\begin{tabular}{|c|c|c|}
\hline elliptic & multiplicative & additive \\
\hline$A_{0}^{(1)}$ & $A_{0}^{(1) *}, A_{1}^{(1)}, A_{2}^{(1)}$, & $A_{0}^{(1) * *}, A_{1}^{(1) *}, A_{2}^{(1) *}$, \\
& $A_{3}^{(1)}, \ldots, A_{6}^{(1)}, A_{7}^{(1)}, A_{7}^{(1) \prime}, A_{8}^{(1)}$ & $D_{4}^{(1)}, D_{5}^{(1)}, D_{6}^{(1)}, D_{7}^{(1)}, D_{8}^{(1)}$, \\
& $E_{6}^{(1)}, E_{7}^{(1)}, E_{8}^{(1)}$ \\
\hline
\end{tabular}

The surfaces of type $A_{8}^{(1)}, D_{8}^{(1)}$, and $E_{8}^{(1)}$ have no Cremona isometries of infinite order. That is, there are no discrete Painlevé equations in these cases. In addition, all the surfaces other than of type $E_{8}^{(1)}$ have a blowingdown to $\mathbb{P}^{1} \times \mathbb{P}^{1}$. In most cases, the image of the anti-canonical divisor can be taken as $f_{0}^{2} g_{0}^{2}=0, f_{0} f_{1} g_{0} g_{1}=0$, or $f_{0} f_{1} g_{0}^{2}=0$ on $\mathbb{P}^{1} \times \mathbb{P}^{1}$, where $\left(f_{0}: f_{1}\right),\left(g_{0}: g_{1}\right)$ are a bi-homogeneous coordinate. The exceptions are of type $A_{2}^{(1) *}, A_{2}^{(1)}, A_{1}^{(1) *}, A_{1}^{(1)}, A_{0}^{(1) * *}, A_{0}^{(1) *}$, and $A_{0}^{(1)}$.

In Sections 2-4, we consider the three regular cases, respectively. We first look at concrete forms of discrete systems and then we write them as discrete Hamiltonian systems. In Section 5, we consider the exceptional cases. We will 
only look at a specific calculation of type $A_{2}^{(1)}$. It should be noted, however, that the discrete Painlevé equations we will see here are nothing but wellknown representatives for each surface and that each surface can have an infinite number of different discrete equations.

\section{The case: ${f_{0}}^{2} g_{0}{ }^{2}=0$}

These are cases where the image of the anti-canonical divisor can be chosen as $f_{0}{ }^{2} g_{0}{ }^{2}=0$. The surfaces of type $D_{5}^{(1)}, D_{6}^{(1)}, D_{7}^{(1)}, E_{6}^{(1)}$, and $E_{7}^{(1)}$ fall into this category. In addition to discrete equations, the differential Painlevé equations arise from these surfaces. Using the inhomogeneous coordinate $f=f_{1} / f_{0}$ and $g=g_{1} / g_{0}$, the Hamiltonians of these differential Painlevé equations are expressible in biquadratic forms:

$$
\begin{gathered}
\frac{\mathrm{d} f}{\mathrm{~d} t}=\frac{\partial H}{\partial g}, \quad \frac{\mathrm{d} g}{\mathrm{~d} t}=-\frac{\partial H}{\partial f} \\
H=\left(g^{2}, g, 1\right)\left(\begin{array}{lll}
m_{22} & m_{21} & m_{20} \\
m_{12} & m_{11} & m_{10} \\
m_{02} & m_{01} & m_{00}
\end{array}\right)\left(\begin{array}{c}
f^{2} \\
f \\
1
\end{array}\right),
\end{gathered}
$$

where the matrix $M=\left(m_{i j}\right)_{i, j=2,1,0}$ can be chosen as follows:

$$
\begin{gathered}
M=M_{D_{5}}=\left(\begin{array}{ccc}
1 & s & 0 \\
1 & s+a_{1}+a_{3} & -a_{2} s \\
0 & a_{1} & 0
\end{array}\right), \quad M_{D_{6}}=\left(\begin{array}{ccc}
1 & 0 & 0 \\
1 & -a_{1}-b_{1} & -s \\
0 & -a_{1} & 0
\end{array}\right), \\
M_{D_{7}}=\left(\begin{array}{ccc}
1 & 0 & 0 \\
0 & a_{1} & s \\
0 & 1 & 0
\end{array}\right), M_{E_{6}}=\left(\begin{array}{ccc}
0 & 1 & 0 \\
-1 & -s & -a_{2} \\
0 & -a_{1} & 0
\end{array}\right), M_{E_{7}}=\left(\begin{array}{ccc}
0 & 0 & 1 \\
-1 & 0 & -s \\
0 & -a_{1} & 0
\end{array}\right) .
\end{gathered}
$$

Discrete Painlevé systems can be expressed in terms of these matrices $M=$ $\left(m_{i j}\right)_{i, j=2,1,0}$ as

$$
g=-\bar{g}-\frac{\widehat{m}_{12} f^{2}+\widehat{m}_{11} f+\widehat{m}_{10}}{\widehat{m}_{22} f^{2}+\widehat{m}_{21} f+\widehat{m}_{20}}, \quad \bar{f}=-f-\frac{\bar{m}_{21} \bar{g}^{2}+\bar{m}_{11} \bar{g}+\bar{m}_{01}}{\bar{m}_{22} \bar{g}^{2}+\bar{m}_{12} \bar{g}+\bar{m}_{02}},
$$

where $\widehat{m}_{i j}$ denotes an intermediate parameter between $m_{i j}$ and $\bar{m}_{i j}$ and the time evolution of the parameters is given by

$$
\begin{array}{ll}
D_{5}: \bar{a}_{1}=a_{1}-1, \bar{a}_{2}=a_{2}+1, \bar{a}_{3}=a_{3}-1, & \left(\widehat{a}_{1}=a_{1}-1, \widehat{a}_{2}=a_{2}, \widehat{a}_{3}=a_{3}\right) \\
D_{6}: \bar{a}_{1}=a_{1}-1, \bar{b}_{1}=b_{1}-1, & \left(\widehat{a}_{1}=a_{1}-1, \widehat{b}_{1}=b_{1}\right) \\
D_{7}: \bar{a}_{1}=a_{1}-2, & \left(\widehat{a}_{1}=a_{1}-1\right) \\
E_{6}: \bar{a}_{1}=a_{1}-1, \bar{a}_{2}=a_{2}+1, & \left(\widehat{a}_{1}=a_{1}, \widehat{a}_{2}=a_{2}\right) \\
E_{7}: \bar{a}_{1}=a_{1}+1, & \left(\widehat{a}_{1}=a_{1}\right) .
\end{array}
$$


Remark 2.1. - The parameters $\left\{\widehat{m}_{i j}\right\}$ in the first equation of (2.3) are slightly different from $\left\{m_{i j}\right\}$. The authors would like to thank an anonymous referee for pointing it out.

Defining the generating function $W$ by

$$
\begin{aligned}
W=W(f, \bar{g})=-f \bar{g}-\int \frac{\widehat{m}_{12} f^{2}+\widehat{m}_{11} f+\widehat{m}_{10}}{\widehat{m}_{22} f^{2}+\widehat{m}_{21} f+\widehat{m}_{20}} \mathrm{~d} f \\
\quad-\int \frac{\bar{m}_{21} \bar{g}^{2}+\bar{m}_{11} \bar{g}+\bar{m}_{01}}{\bar{m}_{22} \bar{g}^{2}+\bar{m}_{12} \bar{g}+\bar{m}_{02}} \mathrm{~d} \bar{g},
\end{aligned}
$$

the discrete system (2.3) is expressed as

$$
g=\frac{\partial W}{\partial f}, \quad \bar{f}=\frac{\partial W}{\partial \bar{g}} .
$$

The explicit forms of these discrete Hamiltonians for each type are

$$
\begin{aligned}
W=W_{D_{5}}= & -f \bar{g}-\bar{g} s-f-\bar{a}_{3} \log (\bar{g}+1)-\bar{a}_{1} \log \bar{g}+a_{2} \log f \\
& \quad\left(a_{1}+a_{2}+a_{3}-1\right) \log (f+s), \\
W_{D_{6}}= & -f \bar{g}-f-\frac{s}{f}+\left(a_{1}+b_{1}-1\right) \log f+\bar{a}_{1} \log \bar{g}+\bar{b}_{1} \log (\bar{g}+1), \\
W_{D_{7}}= & -f \bar{g}+\frac{s}{f}+\frac{1}{\bar{g}}-\left(a_{1}-1\right) \log f-\bar{a}_{1} \log \bar{g} \\
W_{E_{6}}= & -f \bar{g}+\frac{f^{2}}{2}+s f+\frac{\bar{g}^{2}}{2}-s \bar{g}+a_{2} \log f-\bar{a}_{1} \log \bar{g} \\
W_{E_{7}}= & -f \bar{g}+s f+\frac{f^{3}}{3}-\bar{a}_{1} \log \bar{g} .
\end{aligned}
$$

For instance, when the surface is of type $E_{7}^{(1)}$, the discrete Hamiltonian $W_{E_{7}}$ gives the system

$$
\begin{aligned}
& g=\frac{\partial W_{E_{7}}}{\partial f}=-\bar{g}+s+f^{2}, \\
& \bar{f}=\frac{\partial W_{E_{7}}}{\partial \bar{g}}=-f-\frac{\bar{a}_{1}}{\bar{g}},
\end{aligned}
$$

which is in fact a discrete Painlevé system of type $E_{7}^{(1)}$.

\section{The case: $f_{0} f_{1} g_{0} g_{1}=0$}

These are cases when the image of the anti-canonical curve can be chosen as $f_{0} f_{1} g_{0} g_{1}=0$. The surfaces of type $A_{3}^{(1)}, A_{4}^{(1)}, A_{5}^{(1)}, A_{6}^{(1)}, A_{7}^{(1)}, A_{7}^{(1) \prime}$ and $A_{8}^{(1)}$ fall into this category. There are no differential Painlevé equations 
attached to these surfaces and there are no discrete Painleve systems of type $A_{8}^{(1)}$. Discrete Painlevé systems are expressed with a 3 by 3 matrix $M=\left(m_{i j}\right)_{i, j=2,1,0}$ as

$$
g=\frac{\widehat{m}_{02} f^{2}+\widehat{m}_{01} f+\widehat{m}_{00}}{\bar{g}\left(\widehat{m}_{22} f^{2}+\widehat{m}_{21} f+\widehat{m}_{20}\right)}, \quad \bar{f}=\frac{\bar{m}_{20} \bar{g}^{2}+\bar{m}_{10} \bar{g}+\bar{m}_{00}}{f\left(\bar{m}_{22} \bar{g}^{2}+\bar{m}_{12} \bar{g}+\bar{m}_{02}\right)} .
$$

The symplectic form is $\omega=\frac{\mathrm{d} g \wedge \mathrm{d} f}{f g}=\mathrm{d} \log g \wedge \mathrm{d} \log f$. Using $G=\log g$ and $F=\log f$, the system can be expressed as

$$
\begin{aligned}
& G=-\bar{G}+\log \left(\widehat{m}_{02} e^{2 F}+\widehat{m}_{01} e^{F}+\widehat{m}_{00}\right)-\log \left(\widehat{m}_{22} e^{2 F}+\widehat{m}_{21} e^{F}+\widehat{m}_{20}\right), \\
& \bar{F}=-F+\log \left(\bar{m}_{20} e^{2 \bar{G}}+\bar{m}_{10} e^{\bar{G}}+\bar{m}_{00}\right)-\log \left(\bar{m}_{22} e^{2 \bar{G}}+\bar{m}_{12} e^{\bar{G}}+\bar{m}_{02}\right) .
\end{aligned}
$$

Defining the generating function $\widetilde{W}$ by

$$
\begin{aligned}
\widetilde{W}=\widetilde{W}(F, \bar{G})=-F \bar{G}+ & \int \log \left(\widehat{m}_{02} e^{2 F}+\widehat{m}_{01} e^{F}+\widehat{m}_{00}\right) \mathrm{d} F \\
& -\int \log \left(\widehat{m}_{22} e^{2 F}+\widehat{m}_{21} e^{F}+\widehat{m}_{20}\right) \mathrm{d} F \\
& +\int \log \left(\bar{m}_{20} e^{2 \bar{G}}+\bar{m}_{10} e^{\bar{G}}+\bar{m}_{00}\right) \mathrm{d} \bar{G} \\
& -\int \log \left(\bar{m}_{22} e^{2 \bar{G}}+\bar{m}_{12} e^{\bar{G}}+\bar{m}_{02}\right) \mathrm{d} \bar{G},
\end{aligned}
$$

the discrete system (2.3) is written as

$$
G=\frac{\partial \widetilde{W}}{\partial F}, \quad \bar{F}=\frac{\partial \widetilde{W}}{\partial \bar{G}} .
$$

In order to write the system in $f$ and $g$, we introduce $W(f, \bar{g})=\widetilde{W}(\log f, \log \bar{g})$ :

$$
\begin{aligned}
W(f, \bar{g})=-\log f \log \bar{g} & +\int \log \left(\widehat{m}_{02} f^{2}+\widehat{m}_{01} f+\widehat{m}_{00}\right) \frac{\mathrm{d} f}{f} \\
& -\int \log \left(\widehat{m}_{22} f^{2}+\widehat{m}_{21} f+\widehat{m}_{20}\right) \frac{\mathrm{d} f}{f} \\
& +\int \log \left(\bar{m}_{20} \bar{g}^{2}+\bar{m}_{10} \bar{g}+\bar{m}_{00}\right) \frac{\mathrm{d} \bar{g}}{\bar{g}} \\
& -\int \log \left(\bar{m}_{22} \bar{g}^{2}+\bar{m}_{12} \bar{g}+\bar{m}_{02}\right) \frac{\mathrm{d} \bar{g}}{\bar{g}},
\end{aligned}
$$

with which the discrete system (3.1) is expressed as

$$
g=\exp \left(f \frac{\partial W}{\partial f}\right), \quad \bar{f}=\exp \left(\bar{g} \frac{\partial W}{\partial \bar{g}}\right) .
$$


For each type of surface, the matrices $M$ can be chosen as

$$
\begin{aligned}
& M_{A_{3}}=\left(\begin{array}{ccc}
a_{0} a_{5} & -1 /\left(a_{1} a_{2}^{2} a_{3}\right)-a_{0} a_{3} a_{5} & 1 /\left(a_{1} a_{2}^{2}\right) \\
-\left(1+a_{0}\right) a_{5} & 0 & -\left(1+a_{1}\right) / a_{1} a_{2} \\
a_{5} & -1-a_{5} & 1
\end{array}\right), \\
& M_{A_{4}}=\left(\begin{array}{ccc}
0 & 1 & -1 \\
a_{0} / a_{2} & 0 & 1+\left(1 / a_{4}\right) \\
-a_{0} a_{3} / a_{2} & a_{0} a_{3}+\left(1 / a_{2} a_{4}\right) & -1 / a_{4}
\end{array}\right), \\
& M_{A_{5}}=\left(\begin{array}{ccc}
0 & b_{1} / a_{2} & 0 \\
a_{0} & 0 & -b_{1} / a_{2} \\
1 / a_{1} & -1-\left(1 / a_{1}\right) & 1
\end{array}\right), \quad M_{A_{6}}=\left(\begin{array}{ccc}
0 & 1 / b & 0 \\
1 & 0 & -1 / b \\
0 & -a_{1} & a_{1}
\end{array}\right) \text {, } \\
& M_{A_{7}^{\prime}}=\left(\begin{array}{ccc}
1 & -a_{0} & 0 \\
0 & 0 & 0 \\
0 & -1 & 1
\end{array}\right), \quad M_{A_{7}}=\left(\begin{array}{ccc}
0 & -a_{0} & 0 \\
1 & 0 & 0 \\
0 & -1 & 1
\end{array}\right) .
\end{aligned}
$$

The time evolution of the parameters is given by

$$
\begin{aligned}
A_{3}: \bar{a}_{0}=a_{0}, \bar{a}_{1}=a_{1}, & \bar{a}_{2}=q a_{2}, \bar{a}_{3}=a_{3} / q, \\
& \left(\widehat{a}_{0}=a_{0} / q, \widehat{a}_{1}=a_{1}, \widehat{a}_{2}=a_{2}, \widehat{a}_{3}=a_{3}, \widehat{a}_{5}=a_{5}\right) \\
A_{4}: \bar{a}_{0}=a_{0}, \bar{a}_{2}=a_{2} / q, \bar{a}_{3}=q a_{3}, \bar{a}_{4}=a_{4}, & \left(\widehat{a}_{0}=a_{0}, \widehat{a}_{2}=a_{2} / q, \widehat{a}_{3}=a_{3}, \widehat{a}_{4}=a_{4}\right) \\
A_{5}: \bar{a}_{0}=a_{0}, \bar{a}_{1}=a_{1}, & \bar{a}_{2}=a_{2}, \bar{b}_{1}=q b_{1}, \\
& \left(\widehat{a}_{0}=a_{0}, \widehat{a}_{1}=a_{1}, \widehat{a}_{2}=a_{2}, \widehat{b}_{1}=q b_{1}\right)
\end{aligned}
$$

$A_{6}: \bar{a}_{1}=a_{1}, \bar{b}=q b, \quad\left(\widehat{a}_{1}=a_{1}, \widehat{b}=q b\right)$

$A_{7}^{\prime}, A_{7}: \bar{a}_{0}=a_{0} / q, \quad\left(\widehat{a}_{0}=a_{0} / q\right)$.

The corresponding discrete Hamiltonians can be explicitly written as

$$
\begin{aligned}
W=W_{A_{3}}= & -\log f \log \bar{g}+\operatorname{Li}_{2}(\bar{g})+\operatorname{Li}_{2}\left(\bar{a}_{0} \bar{g}\right)-\operatorname{Li}_{2}\left(\frac{\bar{g}}{\bar{a}_{2}}\right)-\operatorname{Li}_{2}\left(\frac{\bar{g}}{\bar{a}_{1} \bar{a}_{2}}\right) \\
& -\operatorname{Li}_{2} f+\operatorname{Li}_{2}\left(\frac{f}{a_{3}}\right)-\operatorname{Li}_{2}\left(a_{5} f\right)+\operatorname{Li}_{2}\left(\frac{a_{0} a_{1} a_{2}^{2} a_{3} a_{5} f}{q}\right) \\
& -\log \bar{a}_{5} \log \bar{g}+\log \left(a_{1} a_{2}{ }^{2}\right) \log f \\
W_{A_{4}}= & -\log f \log \bar{g}+\operatorname{Li}_{2}(f)-\operatorname{Li}_{2}\left(\frac{q f}{a_{2}}\right)-\operatorname{Li}_{2}\left(a_{0} a_{3} a_{4} f\right) \\
& -\operatorname{Li}_{2}(\bar{g})-\operatorname{Li}_{2}\left(\bar{a}_{4} \bar{g}\right)+\operatorname{Li}_{2}\left(\frac{\bar{g}}{\bar{a}_{3}}\right)-\log a_{4} \log f \\
& +\left(\log \frac{\bar{a}_{2}}{\bar{a}_{0} \bar{a}_{3} \bar{a}_{4}}\right) \log \bar{g},
\end{aligned}
$$




$$
\begin{aligned}
W_{A_{5}}= & -\log f \log \bar{g}-\operatorname{Li}_{2}(f)-\operatorname{Li}_{2}\left(\frac{f}{a_{1}}\right)-\operatorname{Li}_{2}\left(\frac{\bar{b}_{1} \bar{g}}{\bar{a}_{2}}\right) \\
& +\operatorname{Li}_{2}\left(-\bar{a}_{0} \bar{a}_{1} \bar{g}\right)-\frac{1}{2}\left(\log \frac{q b_{1} f}{a_{1}}\right)^{2}+\log \bar{a}_{1} \log \bar{g}, \\
W_{A_{6}}= & -\log f \log \bar{g}-\operatorname{Li}_{2}(f)-\operatorname{Li}_{2}\left(\frac{\bar{g}}{\bar{a}_{1} \bar{b}}\right)-\frac{1}{2}\left(\log \frac{f}{q b}\right)^{2} \\
& -\frac{1}{2}(\log \bar{g})^{2}+\log a_{1} \log f+\log \bar{a}_{1} \log \bar{g}, \\
W_{A_{7}^{\prime}}= & -\log f \log \bar{g}-\operatorname{Li}_{2}(f)+\operatorname{Li}_{2}\left(\frac{q f}{a_{0}}\right) \\
& -\frac{1}{2}(\log f)^{2}-(\log \bar{g})^{2}-\log \frac{-a_{0}}{q} \log f, \\
W_{A_{7}}= & -\log f \log \bar{g}-\operatorname{Li}_{2}(f)-\frac{1}{2}\left(\log \frac{-a_{0} f}{q}\right)^{2}-\frac{1}{2}(\log \bar{g})^{2},
\end{aligned}
$$

where $\mathrm{Li}_{2}(x)$ is the dilogarithm function

$$
\operatorname{Li}_{2}(x)=-\int \frac{\log (1-x)}{x} \mathrm{~d} x=\sum_{k=1}^{\infty} \frac{x^{k}}{k^{2}} .
$$

For instance, the discrete Hamiltonian $W_{A_{7}^{\prime}}$ gives rise to

$$
\begin{aligned}
g & =\exp \left(f \frac{\partial W_{A_{7}^{\prime}}}{\partial f}\right) \\
& =\exp \left(-\log \bar{g}-\log f+\log (1-f)-\log \left(1-\frac{q f}{a_{0}}\right)-\log \frac{-a_{0}}{q}\right) \\
& =\frac{1-f}{\bar{g} f\left(f-\frac{a_{0}}{q}\right)}, \\
\bar{f} & =\exp \left(\bar{g} \frac{\partial W_{A_{7}^{\prime}}}{\partial \bar{g}}\right)=\exp (-\log f-2 \log \bar{g})=\frac{1}{f \bar{g}^{2}},
\end{aligned}
$$

which is in fact a discrete Painlevé system of type $A_{7}^{(1) \prime}$.

\section{The case: $f_{0} f_{1} g_{0}^{2}=0$}

These are cases when the image of the anti-canonical curve can be chosen as $f_{0} f_{1} g_{0}^{2}=0$. The surfaces of type $D_{4}^{(1)}, D_{5}^{(1)}, D_{6}^{(1)}, D_{7}^{(1)}$, and $D_{8}^{(1)}$ fall into this category. There are no discrete Painlevé system of type $D_{8}^{(1)}$. Discrete 
Painlevé systems are expressed with a 3 by 3 matrix $M=\left(m_{i j}\right)_{i, j=2,1,0}$ as

$$
g=-\bar{g}-\frac{\widehat{m}_{12} f^{2}+\widehat{m}_{11} f+\widehat{m}_{10}}{\widehat{m}_{22} f^{2}+\widehat{m}_{21} f+\widehat{m}_{20}}, \quad \bar{f}=\frac{\bar{m}_{20} \bar{g}^{2}+\bar{m}_{10} \bar{g}+\bar{m}_{00}}{f\left(\bar{m}_{22} \bar{g}^{2}+\bar{m}_{12} \bar{g}+\bar{m}_{02}\right)} .
$$

Defining the generating function $W$ by

$$
\begin{aligned}
& W=W(f, \bar{g})=- \bar{g} \log f-\int \frac{\widehat{m}_{12} f^{2}+\widehat{m}_{11} f+\widehat{m}_{10}}{\widehat{m}_{22} f^{2}+\widehat{m}_{21} f+\widehat{m}_{20}} \frac{\mathrm{d} f}{f} \\
&+\int \log \left(\bar{m}_{20} \bar{g}^{2}+\bar{m}_{10} \bar{g}+\bar{m}_{00}\right) \mathrm{d} \bar{g} \\
&-\int \log \left(\bar{m}_{22} \bar{g}^{2}+\bar{m}_{12} \bar{g}+\bar{m}_{02}\right) \mathrm{d} \bar{g}
\end{aligned}
$$

the discrete system (4.1) is expressed as

$$
g=f \frac{\partial W}{\partial f}, \quad \bar{f}=\exp \left(\frac{\partial W}{\partial \bar{g}}\right) .
$$

We have already treated the cases $D_{5}^{(1)}, D_{6}^{(1)}$, and $D_{7}^{(1)}$ in the previous section. Therefore, let us consider the remaining case: type of $D_{4}^{(1)}$. The matrix $M$ can be chosen as follows:

$$
M_{D_{4}}=\left(\begin{array}{ccc}
1 & -1-s & s \\
a_{1}+2 a_{2} & -a_{1}-2 a_{2}+(s-1) a_{3}+s a_{4} & -s a_{4} \\
a_{2}\left(a_{1}+a_{2}\right) & 0 & 0
\end{array}\right) .
$$

Note that although the $D_{4}^{(1)}$-type surface also possesses a differential equation, the Hamiltonian (1.1) is not completely the same as the biquadratic one defined by $M_{D_{4}}$ since the canonical variables are not $g$ and $\log f$ but $p=g / f$ and $q=f$. The Hamiltonian in the continuous case is given by $H_{\mathrm{VI}}=\frac{1}{q}\left(q^{2} p^{2}, q p, 1\right) M_{D_{4}}\left(\begin{array}{c}q^{2} \\ q \\ 1\end{array}\right)$.

In the case of $D_{4}^{(1)}$, the time evolution of parameters is given by

$$
\begin{aligned}
& \bar{a}_{0}=a_{0}+1, \quad \bar{a}_{1}=a_{1}, \quad \bar{a}_{2}=a_{2}-1, \quad \bar{a}_{3}=a_{3}+1, \quad \bar{a}_{4}=a_{4}, \\
& \left(\widehat{a}_{0}=a_{0}+1, \quad \widehat{a}_{1}=a_{1}-1, \quad \widehat{a}_{2}=a_{2}, \quad \widehat{a}_{3}=a_{3}, \quad \widehat{a}_{4}=a_{4}\right)
\end{aligned}
$$

and the discrete Hamiltonian is given by

$$
\begin{aligned}
W_{D_{4}}= & -\bar{g} \log f+a_{4} \log f+a_{3} \log (1-f) \\
& -\left(a_{1}+2 a_{2}+a_{3}+a_{4}-1\right) \log (1-f / s)+\bar{g}(\log \bar{g}+\log s) \\
& -\left(\bar{g}+\bar{a}_{1}+\bar{a}_{2}\right) \log \left(\bar{g}+\bar{a}_{1}+\bar{a}_{2}\right)-\left(\bar{g}+\bar{a}_{2}\right) \log \left(\bar{g}+\bar{a}_{2}\right) \\
& +\left(\bar{g}-\bar{a}_{4}\right) \log \left(\bar{g}-\bar{a}_{4}\right) .
\end{aligned}
$$


The discrete system determined by $W_{D_{4}}$ is

$$
\begin{aligned}
g & =f \frac{\partial W_{D_{4}}}{\partial f}=-\bar{g}+a_{4}-\frac{a_{3} f}{1-f}+\frac{\left(a_{1}+2 a_{2}+a_{3}+a_{4}-1\right) f}{s-f} \\
& =-\bar{g}+1-a_{1}-2 a_{2}-\frac{a_{3}}{1-f}+\frac{a_{1}+2 a_{2}+a_{3}+a_{4}-1}{1-f / s} \\
\bar{f} & =\exp \left(\frac{\partial W_{D_{4}}}{\partial \bar{g}}\right) \\
& =\exp \left(-\log f+\log \bar{g}+\log \left(\bar{g}-\bar{a}_{4}\right)+\log s-\log \left(\bar{g}+\bar{a}_{1}+\bar{a}_{2}\right)-\log \left(\bar{g}+\bar{a}_{2}\right)\right) \\
& =\frac{s \bar{g}\left(\bar{g}-\bar{a}_{4}\right)}{f\left(\bar{g}+\bar{a}_{1}+\bar{a}_{2}\right)\left(\bar{g}+\bar{a}_{2}\right)},
\end{aligned}
$$

which is in fact a discrete Painlevé system of type $D_{4}^{(1)}$.

\section{The other cases}

The surfaces of type $A_{2}^{(1) *}, A_{2}^{(1)}, A_{1}^{(1) *}, A_{1}^{(1)}, A_{0}^{(1) * *}, A_{0}^{(1) *}$, and $A_{0}^{(1)}$ fall into this category. Elliptic difference systems arise for the $A_{0}^{(1)}$-type surface but, at this moment, a discrete Hamiltonian is difficult to write down for some technical reasons. Therefore, we do not consider the case $A_{0}^{(1)}$.

In the other cases, we can take $F=F(f, g), G=G(f, g)$ so that the symplectic form can be expressed as $\omega=\mathrm{d} \log G \wedge \mathrm{d} \log F$ or $\mathrm{d} G \wedge \mathrm{d} \log F$. Discrete Painlevé systems are expressed by a generating function $W=W(F, \bar{G})$ as

$$
G=F \frac{\partial W}{\partial F}, \quad \bar{F}=\exp \left(\frac{\partial W}{\partial \bar{G}}\right)
$$

or

$$
G=\exp \left(F \frac{\partial W}{\partial F}\right), \quad \bar{F}=\exp \left(\bar{G} \frac{\partial W}{\partial \bar{G}}\right) .
$$

Such a $W$ is an algebraic function in $F$ and $\bar{G}$ but does not have concise expression since it is not single valued.

However, if the system has the form $g=\varphi(f, \bar{g}), \bar{f}=\psi(f, \bar{g}), \widetilde{W}$ has a concise expression in $f, \bar{g}$. Here, we use the notation $\widetilde{W}(f, \bar{g})=W(F(f, \psi(f, \bar{g}))$, $\bar{G}(\varphi(f, \bar{g}), \bar{g}))$. Nevertheless, even with $\widetilde{W}(f, \bar{g})$, the expressions are not as simple as we want.

While the additive type surfaces $A_{2}^{(1) *}, A_{1}^{(1) *}$, and $A_{0}^{(1) * *}$ correspond to (5.1), the multiplicative type surfaces $A_{2}^{(1)}, A_{1}^{(1)}$, and $A_{0}^{(1) *}$ correspond 
to (5.2). Let us consider the function $\widetilde{W}$ for the multiplicative surface of type $A_{2}^{(1)}$. One of the discrete systems of type $A_{2}^{(1)}$ is

$$
\begin{aligned}
q-P\left(A_{2}\right): & \left(\begin{array}{llll}
b_{1} & b_{2} & b_{3} & b_{4} \\
b_{5} & b_{6} & b_{7} & b_{8}
\end{array} ;, g\right) \mapsto\left(\begin{array}{cccc}
b_{1} & b_{2} & b_{3} & b_{4} \\
q b_{5} & q b_{6} & q b_{7} & q b_{8}
\end{array} \bar{f}, \bar{g}\right), \\
& \frac{(f g-1)(f \bar{g}-1)}{q b_{7} b_{8}}=\frac{\left(f-b_{1}\right)\left(f-b_{2}\right)\left(f-b_{3}\right)\left(f-b_{4}\right)}{\left(f-b_{5}\right)\left(f-b_{6}\right)}, \\
& \frac{(f \bar{g}-1)(\bar{f} \bar{g}-1)}{q b_{5} b_{6}}=\frac{\left(\bar{g}-1 / b_{1}\right)\left(\bar{g}-1 / b_{2}\right)\left(\bar{g}-1 / b_{3}\right)\left(\bar{g}-1 / b_{4}\right)}{\left(\bar{g}-q b_{7}\right)\left(\bar{g}-q b_{8}\right)}, \\
& \left(q=\frac{b_{5} b_{6}}{b_{1} b_{2} b_{3} b_{4} b_{7} b_{8}}\right) .
\end{aligned}
$$

The symplectic form is $\omega=\mathrm{d} \log G \wedge \mathrm{d} \log F$, where $F=f g-1, G=g$. Putting

$$
\begin{aligned}
\varphi(f, \bar{g}) & =\frac{1}{f}\left(1+q b_{7} b_{8} \frac{\left(f-b_{1}\right)\left(f-b_{2}\right)\left(f-b_{3}\right)\left(f-b_{4}\right)}{(f \bar{g}-1)\left(f-b_{5}\right)\left(f-b_{6}\right)}\right), \\
\psi(f, \bar{g}) & =\frac{1}{\bar{g}}\left(1+q b_{5} b_{6} \frac{\left(\bar{g}-1 / b_{1}\right)\left(\bar{g}-1 / b_{2}\right)\left(\bar{g}-1 / b_{3}\right)\left(\bar{g}-1 / b_{4}\right)}{(f \bar{g}-1)\left(\bar{g}-q b_{7}\right)\left(\bar{g}-q b_{8}\right)}\right),
\end{aligned}
$$

$\widetilde{W}(f, \bar{g})=W(f \varphi(f, \bar{g})-1, \bar{g})$ satisfies

$$
\begin{aligned}
& \frac{\partial \widetilde{W}}{\partial f}=\frac{\partial W}{\partial F} \frac{\partial F}{\partial f}=\left(\varphi+f \frac{\partial \varphi}{\partial f}\right) \frac{\partial W}{\partial F}, \\
& \frac{\partial \widetilde{W}}{\partial \bar{g}}=\frac{\partial W}{\partial F} \frac{\partial F}{\partial \bar{g}}+\frac{\partial W}{\partial \bar{G}}=f \frac{\partial \varphi}{\partial \bar{g}} \frac{\partial W}{\partial F}+\frac{\partial W}{\partial \bar{G}} .
\end{aligned}
$$

Therefore, we have

$$
\begin{aligned}
& \varphi=\exp \left(F \frac{\partial W}{\partial F}\right)=\exp \left(\frac{f \varphi-1}{\varphi+f \frac{\partial \varphi}{\partial f}} \frac{\partial \widetilde{W}}{\partial f}\right) \\
& \psi=\frac{1+\exp \left(\bar{G} \frac{\partial W}{\partial \bar{G}}\right)}{\bar{G}}=\frac{1}{\bar{g}}\left(1+\exp \left(\bar{g} \frac{\partial \widetilde{W}}{\partial \bar{g}}-\frac{f \bar{g} \frac{\partial \varphi}{\partial \bar{g}}}{\varphi+f \frac{\partial \varphi}{\partial f}} \frac{\partial \widetilde{W}}{\partial f}\right)\right) .
\end{aligned}
$$

By integrating

$$
\frac{\partial \widetilde{W}}{\partial f}=\frac{\varphi+f \frac{\partial \varphi}{\partial f}}{f \varphi-1} \log \varphi, \quad \frac{\partial \widetilde{W}}{\partial \bar{g}}=\frac{1}{\bar{g}} \log (\bar{g} \psi-1)+\frac{f \frac{\partial \varphi}{\partial \bar{g}}}{f \varphi-1} \log \varphi,
$$


we obtain $\widetilde{W}=\widetilde{W}_{A_{2}}$ as

$$
\begin{aligned}
\widetilde{W}_{A_{2}}= & \operatorname{Li}_{2}(f \bar{g})+\operatorname{Li}_{2}\left(\frac{\left(1-f / b_{1}\right)\left(1-f / b_{2}\right)\left(1-f / b_{3}\right)\left(1-f / b_{4}\right)}{(1-f \bar{g})\left(1-f / b_{5}\right)\left(1-f / b_{6}\right)}\right) \\
& +\log f \log (1-f \bar{g})+\sum_{k=1}^{4} \operatorname{Li}_{2}\left(1-\frac{f}{b_{k}}\right)-\sum_{l=5,6} \operatorname{Li}_{2}\left(1-\frac{f}{b_{l}}\right) \\
& +\sum_{k=1}^{4} \log b_{k} \log \left(1-\frac{f}{b_{k}}\right)-\sum_{l=5,6} \log b_{l} \log \left(1-\frac{f}{b_{l}}\right) \\
& -\sum_{k=1}^{4} \operatorname{Li}_{2}\left(b_{k} \bar{g}\right)+\sum_{l=7,8} \operatorname{Li}_{2}\left(\frac{\bar{g}}{q b_{l}}\right)+\pi \sqrt{-1} \log \bar{g} .
\end{aligned}
$$

Using $\widetilde{W}$, the discrete system (5.3) is expressed as

$$
\begin{aligned}
& g=\exp \left(-\left(\sum_{k=1}^{4} \frac{1 / b_{k}}{1-f / b_{k}}-\sum_{l=5,6} \frac{1 / b_{l}}{1-f / b_{l}}-\frac{\bar{g}}{1-f \bar{g}}\right)^{-1} \frac{\partial \widetilde{W}}{\partial f}\right), \\
& \bar{f}=\frac{1}{\bar{g}}\left(1+\exp \left(\bar{g} \frac{\partial \widetilde{W}}{\partial \bar{g}}-\frac{f \bar{g}}{1-f \bar{g}} \frac{\partial \widetilde{W}}{\partial f}\right)\right)
\end{aligned}
$$

which is, however, probably not a satisfactory answer one hopes.

For the other cases, let us merely write coordinates $F$ and $G$. In the cases of type $A_{2}^{(1) *}, A_{1}^{(1) *}$, and $A_{0}^{(1) * *}$, the coordinates

$$
\begin{aligned}
& A_{2}^{(1) *}:(F, G)=(f+g, g), \quad A_{1}^{(1) *}:(F, G)=\left(1-\frac{2 r}{f+g}, g\right), \\
& A_{0}^{(1) * *}:(F, G)=\left((f-g)^{2}-8 r^{2}(f+g)+16 r^{4}, f-g\right)
\end{aligned}
$$

give the symplectic form $\mathrm{d} G \wedge \mathrm{d} \log F$. For the surfaces of type $A_{2}^{(1)}, A_{1}^{(1)}$, and $A_{0}^{(1) *}$, the coordinates

$$
\begin{gathered}
A_{2}^{(1)}:(F, G)=(f g-1, g), \quad A_{1}^{(1)}:(F, G)=\left(1-\frac{r^{2}-1}{f g-1}, g\right), \\
A_{0}^{(1) *}:(F, G)=\left((f+g)^{2}-\left(r^{2}+\frac{1}{r^{2}}\right) f g+r^{2}-\frac{1}{r^{2}},-\frac{f}{r}+r g\right)
\end{gathered}
$$

give the symplectic form $\mathrm{d} \log G \wedge \mathrm{d} \log F$.

\section{Bibliography}

[1] B. GAmBiER, "Sur les équations différentielles du second ordre et du premier degré dont l'intégrale générale est a points critiques fixes", Acta Math. 33 (1909), p. 1-55. 
[2] B. Grammaticos \& A. Ramani, "The hunting for the discrete Painlevé equations", Regul. Chaotic Dyn. 5 (2000), no. 1, p. 53-66.

[3] B. Grammaticos, A. Ramani \& V. Papageorgiou, "Do integrable mappings have the Painlevé property?", Phys. Rev. Lett. 67 (1991), no. 14, p. 1825-1828.

[4] K. Kajiwara, M. Noumi \& Y. Yamada, "Geometric aspects of Painlevé equations", J. Phys. A, Math. Theor. 50 (2017), no. 7, article no. 073001 (164 pages).

[5] Y. Ohta, A. Ramani \& B. Grammaticos, "Elliptic discrete Painlevé equations", J. Phys. A, Math. Gen. 35 (2002), no. 45, p. L653-L659.

[6] P. Painlevé, "Sur les équations différentielles du second ordre et d'ordre supérieur dont l'intégrale générale est uniforme", Acta Math. 25 (1901), p. 1-85.

[7] G. R. W. Quispel, J. A. G. Roberts \& C. J. Thompson, "Integrable mappings and soliton equations II", Physica D 34 (1989), no. 1-2, p. 183-192.

[8] A. Ramani, B. Grammaticos \& J. Hietarinta, "Discrete versions of the Painlevé equations", Phys. Rev. Lett. 67 (1991), no. 14, p. 1829-1832.

[9] H. SAKAI, "Rational surfaces associated with affine root systems and geometry of the Painlevé equations", Commun. Math. Phys. 220 (2001), no. 1, p. 165-229.

[10] A. P. Veselov, "Integrable mappings", Usp. Mat. Nauk 46 (1991), no. 5, p. 3-45, translation in Russian Math. Surveys 46 (1991): no. 5, p. 1-51. 